\title{
Distribution and Determinants of Vitamin D-Binding Protein, Total, "Non-Bioavailable", Bioavailable, and Free 25-Hydroxyvitamin D Concentrations among Older Adults
}

\author{
Anna Zhu ${ }^{1,2}$, Sabine Kuznia ${ }^{1,2}$, Tobias Niedermaier ${ }^{1} \mathbb{D}^{D}$, Bernd Holleczek ${ }^{3}$, Ben Schöttker ${ }^{1,4} \mathbb{D}$ \\ and Hermann Brenner 1,4,5,6,*(D)
}

check for

updates

Citation: Zhu, A.; Kuznia, S.;

Niedermaier, T.; Holleczek, B.;

Schöttker, B.; Brenner, H. Distribution and Determinants of Vitamin D-Binding Protein, Total, "Non-Bioavailable", Bioavailable, and Free 25-Hydroxyvitamin D Concentrations among Older Adults. Nutrients 2021, 13, 3982. https:// doi.org/10.3390/nu13113982

Academic Editor: Tyler Barker

Received: 20 September 2021 Accepted: 5 November 2021 Published: 9 November 2021

Publisher's Note: MDPI stays neutral with regard to jurisdictional claims in published maps and institutional affiliations.

Copyright: (c) 2021 by the authors. Licensee MDPI, Basel, Switzerland. This article is an open access article distributed under the terms and conditions of the Creative Commons Attribution (CC BY) license (https:/ / creativecommons.org/licenses/by/ $4.0 /)$.
1 Division of Clinical Epidemiology and Aging Research, German Cancer Research Center (DKFZ), 69120 Heidelberg, Germany; anna.zhu@dkfz-heidelberg.de (A.Z.); s.kuznia@dkfz-heidelberg.de (S.K.); t.niedermaier@dkfz-heidelberg.de (T.N.); b.schoettker@dkfz-heidelberg.de (B.S.)

2 Medical Faculty Heidelberg, Heidelberg University, 69120 Heidelberg, Germany

3 Saarland Cancer Registry, 66119 Saarbrücken, Germany; b.holleczek@gbe-ekr.saarland.de

4 Network Aging Research, Heidelberg University, 69115 Heidelberg, Germany

5 Division of Preventive Oncology, German Cancer Research Center (DKFZ) and National Center for Tumor Diseases (NCT), 69120 Heidelberg, Germany

6 German Cancer Consortium (DKTK), German Cancer Research Center (DKFZ), 69120 Heidelberg, Germany

* Correspondence: h.brenner@dkfz-heidelberg.de; Tel.: +49-622-142-1300

\begin{abstract}
Background: serum 25-hydroxyvitamin D (25(OH)D) ("total $25 \mathrm{OH}(\mathrm{D})$ ") is the most commonly used indicator of vitamin D status. However, $25(\mathrm{OH}) \mathrm{D}$ is mostly bound to the vitamin $\mathrm{D}$ binding protein (VDBP) or albumin in blood, and it has been suggested that the remaining bioavailable or free $25(\mathrm{OH}) \mathrm{D}$ may be more relevant for vitamin $\mathrm{D}$ associated health outcomes. We aimed to explore distributions and determinants of VDBP, total, bioavailable, complementary "non-bioavailable", and free 25(OH)D in a large cohort of older adults in Germany. Methods: total $25(\mathrm{OH}) \mathrm{D}, \mathrm{VDBP}$, and albumin concentrations were measured in blood samples of 5899 men and women aged 50-75 years and used to calculate bioavailable (and complementary "non-bioavailable") and free $25(\mathrm{OH}) \mathrm{D}$ concentrations. Linear regression models were used to evaluate associations of potential determinants of the various vitamin D biomarkers. Results: mean concentrations of VDBP, total, non-bioavailable, bioavailable, and free 25(OH)D were $323.6 \mu \mathrm{g} / \mathrm{mL}, 49.8 \mathrm{nmol} / \mathrm{L}, 43.4 \mathrm{nmol} / \mathrm{L}$, $2.5 \mathrm{ng} / \mathrm{mL}$, and $5.7 \mathrm{pg} / \mathrm{mL}$, respectively. Seasonal variations were observed for all markers, with peak values in spring for VDBP and in summer for total, non-bioavailable, bioavailable, and free 25(OH)D. Consistent inverse associations were seen with age and body mass index for all markers, but divergent associations were seen with $\mathrm{C}$-reactive protein. Strong variations by VDBP genotypes were seen for bioavailable and free $25(\mathrm{OH}) \mathrm{D}$, and, in opposite direction for non-bioavailable $25(\mathrm{OH}) \mathrm{D}$. Conclusion: commonalities and differences in determinants of various markers of vitamin D status were observed, which may help to enable a better understanding of their potential role for various vitamin D related health outcomes.
\end{abstract}

Keywords: vitamin D-binding protein; bioavailable 25(OH)D; free 25(OH)D; determinants

\section{Introduction}

Vitamin D deficiency is common and closely related to various health outcomes among older adults [1]. The most widely used indicator of vitamin D status is serum 25-hydroxyvitamin D $(25(\mathrm{OH}) \mathrm{D})$. It has been suggested, however, that other vitamin $\mathrm{D}$ biomarkers may be more relevant with respect to health outcomes [2]. About $85-90 \%$ of serum 25(OH)D are bound to vitamin D-binding protein (VDBP) [3]. Serum 25(OH)D that is not bound to VDBP is known as bioavailable $25(\mathrm{OH}) \mathrm{D}$. Around $10-15 \%$ of serum $25(\mathrm{OH}) \mathrm{D}$ are loosely bound to albumin, and less than $1 \%$ is in a free form, known as free $25(\mathrm{OH}) \mathrm{D}[4,5]$. 
Although a number of studies have addressed distribution and potential determinants of the various vitamin D biomarkers in different populations [2,3,6-8], evidence from large-scale epidemiological studies among older adults has remained sparse, mostly descriptive without multivariate adjustments [3,8], or limited to single specific vitamin $\mathrm{D}$ biomarkers [7]. The aim of this study was to assess distributions and determinants of VDBP, total, bioavailable (and complementary "non-bioavailable"), and free 25(OH)D concentrations in a large population cohort of older adults in Germany.

\section{Materials and Methods}

\subsection{Study Design and Population}

Our analysis is based on baseline data from the ESTHER study (German: Epidemiologische Studie zu Chancen der Verhütung, Früherkennung und optimierten Therapie chronischer Erkrankungen in der älteren Bevölkerung), a population-based cohort study of older adults conducted statewide in Saarland, a federal state in Southwestern Germany. Details of the study design have been reported elsewhere [9]. Briefly, 9940 men and women aged 50-75 years were recruited by over 400 general practitioners between 2000 and 2002 during a routine health checkup offered in the German healthcare system. The ESTHER study population has been found to be representative of the German older population with respect to sociodemographic characteristics, lifestyle factors, and health conditions [10]. The current analysis was restricted to 5899 participants recruited in 2001 and 2002 for whom measurements of total $25(\mathrm{OH}) \mathrm{D}$, VDBP, genetic markers, and the necessary covariates needed to derive bioavailable and free $25(\mathrm{OH}) \mathrm{D}$ were available (for details see below).

\subsection{Data and Blood Sample Collection}

We collected information on covariates including age, sex, education, smoking status, physical activity, intake of multivitamin supplements, and fish consumption by using a comprehensive questionnaire at baseline. General practitioners assessed height, weight, and systolic blood pressure, and provided information on presence of hypertension, diabetes, chronic kidney diseases (CKD), and cardiovascular diseases (CVD) at the time of the health check-up. For our analyses, history of CVD was defined as history of coronary heart disease, myocardial infarction, stroke, or stent/balloon catheter operation for revascularization of coronary arteries. We obtained information on previous incident malignant cancer (excluding non-melanoma skin cancer) through record linkage with the long-standing statewide Saarland Cancer Registry.

Blood samples were collected at baseline by general practitioners, centrifuged, shipped to the study center, and stored at $-80{ }^{\circ} \mathrm{C}$ until analysis. Baseline laboratory analyses included total cholesterol (measured by enzymatic chromatography), C-reactive protein (CRP, measured by turbidimetry), and albumin (measured by fluorescence immunoassay).

\subsection{VDBP, Total, Non-Bioavailable, Bioavailable, and Free 25(OH)D Measurements}

From the blood samples collected at baseline, we further measured concentrations of total $25(\mathrm{OH}) \mathrm{D}$ and VDBP. Total $25(\mathrm{OH}) \mathrm{D}$ was measured as the sum of $25(\mathrm{OH}) \mathrm{D}_{2}$ and $25(\mathrm{OH}) \mathrm{D}_{3}$ in the context of two different research projects for women and men. In a first project among women conducted in 2006, total 25(OH)D concentrations were measured by using the DiaSorin-Liaison analyzer (DiaSorin Inc., Stillwater, MN, USA). In a subsequent project among men conducted in 2009, the DiaSorin-Liaison method was no longer available, and total 25(OH)D was measured using IDS-iSYS (Immunodiagnostic Systems $\mathrm{GmbH}$, Frankfurt Main, Germany). Later both immunoassays were standardized to the gold standard method of liquid chromatography tandem mass spectrometry. Details of the laboratory methods for measuring total $25(\mathrm{OH}) \mathrm{D}$ and their standardization have been reported elsewhere [11] and are summarized in the Supplementary File S1. In 2019, VDBP concentrations were measured by enzyme immunoassay (Immundiagnostik Inc., Bensheim, Germany). The intra-assay and inter-assay coefficients of variations were less than $10 \%$. In 
addition, we extracted genetic data of single nucleotide polymorphisms (SNPs) rs7041 and rs4588 for coding VDBP genotypes from genome-wide genotyping of DNA from whole blood samples using the Illumina Infinium OncoArray and Global Screening Array BeadChip (Illumina, San Diego, CA, USA). More details on genotyping procedures including quality control and imputation have been reported elsewhere [12] and are summarized in the Supplementary File S1.

Bioavailable and free $25(\mathrm{OH}) \mathrm{D}$ concentrations were calculated based on the levels of total $25(\mathrm{OH}) \mathrm{D}$, VDBP, albumin, and their affinity constants derived from the VDBP genotypes $[13,14]$. We used the following equation for calculating free $25(\mathrm{OH}) \mathrm{D}$ concentrations:

$$
D_{\text {free }}=\left(-b+\sqrt{b^{2}-4 a c}\right) \div 2 \mathrm{a}
$$

where $\mathrm{a}=\mathrm{K}_{\mathrm{VDBP}} \cdot \mathrm{K}_{\mathrm{alb}} \cdot \mathrm{D}_{\mathrm{alb}}+\mathrm{K}_{\mathrm{VDBP}} ; \mathrm{b}=\mathrm{K}_{\mathrm{VDBP}} \cdot \mathrm{D}_{\mathrm{VDBP}}-\mathrm{K}_{\mathrm{VDBP}} \cdot \mathrm{D}_{\text {total }}+\mathrm{K}_{\mathrm{alb}} \cdot \mathrm{D}_{\mathrm{alb}}+1$; $\mathrm{c}=-\mathrm{D}_{\text {total }}$, and $\mathrm{D}_{\text {free }}$ indicates free $25(\mathrm{OH}) \mathrm{D}$ concentrations; $\mathrm{D}_{\text {alb }}$ indicates albumin concentrations; $\mathrm{D}_{\text {total }}$ indicates total 25(OH)D concentrations; $\mathrm{D}_{\mathrm{VDBP}}$ indicates VDBP concentrations; $\mathrm{K}_{\mathrm{alb}}$ is the affinity constant between vitamin $\mathrm{D}$ and albumin $\left(\mathrm{K}_{\mathrm{alb}}=6 \times 10^{5} \mathrm{M}^{-1}\right)$; $\mathrm{K}_{\mathrm{VDBP}}$ is the affinity constant between vitamin D and VDBP $\left(\mathrm{K}_{\mathrm{VDBP}}=1.12 \times 10^{9} \mathrm{M}^{-1}\right.$ for GC1f-1f; $K_{V D B P}=8.6 \times 10^{8} \mathrm{M}^{-1}$ for GC1f-1s; $K_{V D B P}=7.4 \times 10^{8} \mathrm{M}^{-1}$ for GC1f-2; $\mathrm{K}_{\mathrm{VDBP}}=6.0 \times 10^{8} \mathrm{M}^{-1}$ for GC1s-1s; $\mathrm{K}_{\mathrm{VDBP}}=4.8 \times 10^{8} \mathrm{M}^{-1}$ for GC1s-2; $\mathrm{K}_{\mathrm{VDBP}}=3.6 \times 10^{8} \mathrm{M}^{-1}$ for GC2-2). All concentrations are expressed in $\mathrm{mol} / \mathrm{L}$ in calculating equations.

We calculated bioavailable $25(\mathrm{OH}) \mathrm{D}$ concentrations by using the following equation:

$$
D_{\text {bioavailable }}=D_{\text {free }}+D_{\text {alb }}=\left(K_{\text {alb }} \cdot D_{\text {alb }}+1\right) \cdot D_{\text {free }}
$$

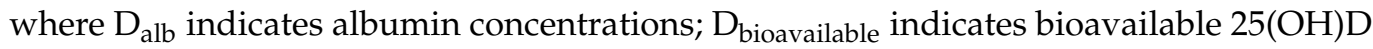
concentrations; $\mathrm{D}_{\text {free }}$ indicates free $25(\mathrm{OH}) \mathrm{D}$ concentrations; $\mathrm{K}_{\mathrm{alb}}$ is the affinity constant between vitamin $\mathrm{D}$ and albumin $\left(\mathrm{K}_{\mathrm{alb}}=6 \times 10^{5} \mathrm{M}^{-1}\right)$. All concentrations are expressed in $\mathrm{mol} / \mathrm{L}$ in calculating equations.

We further calculated "non-bioavailable $25(\mathrm{OH}) \mathrm{D}$ " concentrations as the difference between total and bioavailable $25(\mathrm{OH}) \mathrm{D}$ concentrations.

\subsection{Statistical Analysis}

We summarized the baseline characteristics by using descriptive statistics. Additionally, we visualized the distributions of VDBP, total, non-bioavailable, bioavailable, and free $25(\mathrm{OH}) \mathrm{D}$ concentrations by sex. We plotted histograms and density curves to show the general distribution of these vitamin D biomarkers, and further plotted bar charts to assess potential variations by season and month of blood draw, and VDBP genotypes. We also calculated Spearman's rank correlation coefficients and plotted the correlation analysis of VDBP, total, non-bioavailable, bioavailable, and free 25(OH)D.

We report mean concentrations of VDBP, total, non-bioavailable, bioavailable, and free $25(\mathrm{OH}) \mathrm{D}$ by various demographic, behavioral, and medical characteristics. We used linear regression models to assess individual and independent associations of these factors with vitamin D biomarkers. Using linear regression was judged to be appropriate because all outcomes were approximately normally distributed despite some right skewedness for total and non-bioavailable $25(\mathrm{OH}) \mathrm{D}$ among females. Overall, 783 participants had at least one missing value in one or more of the included covariates (13.3\%). To minimize potential bias, we applied multiple-imputation and report pooled regression results from 20 imputed databases. All analyses were carried out by R software (version: 3.6.2, R Core Team, R Foundation for Statistical Computing, Vienna, Austria). Statistical significance was defined by $p<0.05$ in two-sided testing. 


\subsection{Ethics Statement}

The ESTHER study was approved by the Ethics Committees of the Medical Faculty of the University of Heidelberg and of the Physicians' Board of Saarland. All participants gave written informed consent.

\section{Results}

Table 1 shows the baseline characteristics of 5899 included participants. Mean age was 62.3 (standard deviation (SD): 6.6 ) years, and $43.9 \%$ were males. Approximately half (50.7\%) were never smokers, $32.0 \%, 14.4 \%$, and $67.0 \%$ reported moderate or high physical activity, regular intake of multivitamin supplements, and fish consumption at least once per week, respectively. History of hypertension, diabetes, CVD, cancer, and CKD, were reported for $43.3 \%, 15.0 \%, 19.7 \%, 6.3 \%$, and $8.3 \%$ of participants, respectively. The mean body mass index (BMI), systolic blood pressure, total cholesterol, and CRP was 27.7 (SD: 4.4) $\mathrm{kg} / \mathrm{m}^{2}$, 139.9 (SD: 19.6) mmHg, 230.9 (SD: 42.2) $\mathrm{mg} / \mathrm{dL}$, and 4.2 (SD: 8.1) mg/L, respectively.

Table 1. Baseline characteristics of the study participants $(\mathrm{N}=5899)$.

\begin{tabular}{|c|c|c|}
\hline Characteristic & Mean (SD) & $\mathbf{N}(\%)$ \\
\hline Age (years) & $62.3(6.6)$ & \\
\hline$<60$ & & $1972(33.4)$ \\
\hline $60-64$ & & $1589(26.9)$ \\
\hline $65-69$ & & $1392(23.6)$ \\
\hline$\geq 70$ & & $946(16.0)$ \\
\hline Sex, male & & $2589(43.9)$ \\
\hline \multicolumn{3}{|l|}{ School education } \\
\hline$\leq 9$ years & & $4298(74.8)$ \\
\hline $10-11$ years & & $800(13.9)$ \\
\hline$\geq 12$ years & & $645(11.2)$ \\
\hline \multicolumn{3}{|l|}{ Smoking status } \\
\hline Never smokers & & $2904(50.7)$ \\
\hline Former smokers & & $1871(32.7)$ \\
\hline Current smokers & & $949(16.6)$ \\
\hline Moderate/high physical activity & & $1883(32.0)$ \\
\hline Regular intake of multivitamin supplements & & $831(14.4)$ \\
\hline Fish consumption $(\geq 1$ time/week) & & $3714(67.0)$ \\
\hline Hypertension & & $2545(43.3)$ \\
\hline Diabetes & & $873(15.0)$ \\
\hline Cardiovascular disease & & $1161(19.7)$ \\
\hline Cancer & & $370(6.3)$ \\
\hline Chronic kidney disease & & $491(8.3)$ \\
\hline Body mass index $\left(\mathrm{kg} / \mathrm{m}^{2}\right)$ & $27.7(4.4)$ & \\
\hline Systolic blood pressure (mm Hg) & $139.9(19.6)$ & \\
\hline Total cholesterol $(\mathrm{mg} / \mathrm{dL})$ & $230.9(42.2)$ & \\
\hline C-reactive protein $(\mathrm{mg} / \mathrm{L})$ & $4.2(8.1)$ & \\
\hline
\end{tabular}

$\overline{\mathrm{N}}(\%)$ refers to the original data without imputation. Abbreviations: SD: standard deviation.

Overall mean concentrations of VDBP, total, non-bioavailable, bioavailable, and free $25(\mathrm{OH}) \mathrm{D}$ were $323.6 \mu \mathrm{g} / \mathrm{mL}, 49.8 \mathrm{nmol} / \mathrm{L}, 43.4 \mathrm{nmol} / \mathrm{L}, 2.5 \mathrm{ng} / \mathrm{mL}$, and $5.7 \mathrm{pg} / \mathrm{mL}$, respectively. Figure 1 shows distribution plots of VDBP, total, non-bioavailable, bioavailable, and free 25(OH)D concentrations by sex. For VDBP, a close to normal distribution was observed; the distribution of the other vitamin D biomarkers was right-skewed to a moderate extent. 

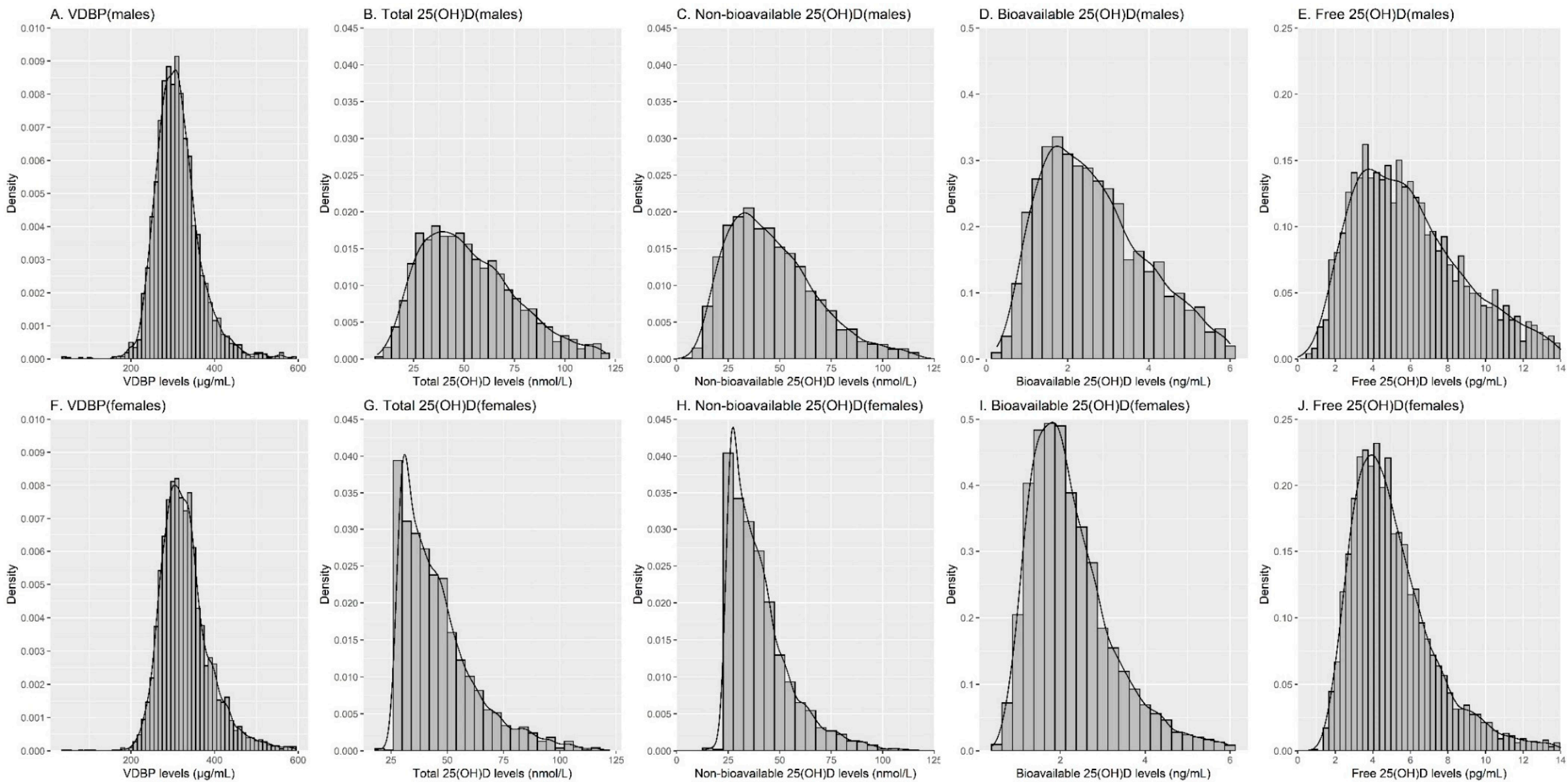

Figure 1. Histograms and density curves of VDBP, total, non-bioavailable, bioavailable, and free 25(OH)D concentrations by sex. Abbreviations: VDBP: vitamin D binding protein. 
Figure 2 presents the correlation matrix for VDBP, total, non-bioavailable, bioavailable, and free $25(\mathrm{OH}) \mathrm{D}$. VDBP concentrations were weakly correlated with total and nonbioavailable $25(\mathrm{OH}) \mathrm{D}$, and moderate negative correlations with bioavailable and free 25(OH)D concentrations were observed (Spearman's rank correlation coefficients: 0.05, $0.11,-0.30$, and -0.32 , respectively). Total $25(\mathrm{OH}) \mathrm{D}$ concentrations were highly correlated with non-bioavailable, bioavailable, and free $25(\mathrm{OH}) \mathrm{D}$ concentrations (Spearman's rank correlation coefficients were $0.99,0.78$, and 0.79 , respectively). Bioavailable $25(\mathrm{OH}) \mathrm{D}$ levels were also strongly correlated with free 25(OH)D levels (Spearman's rank correlation coefficient: 0.99).

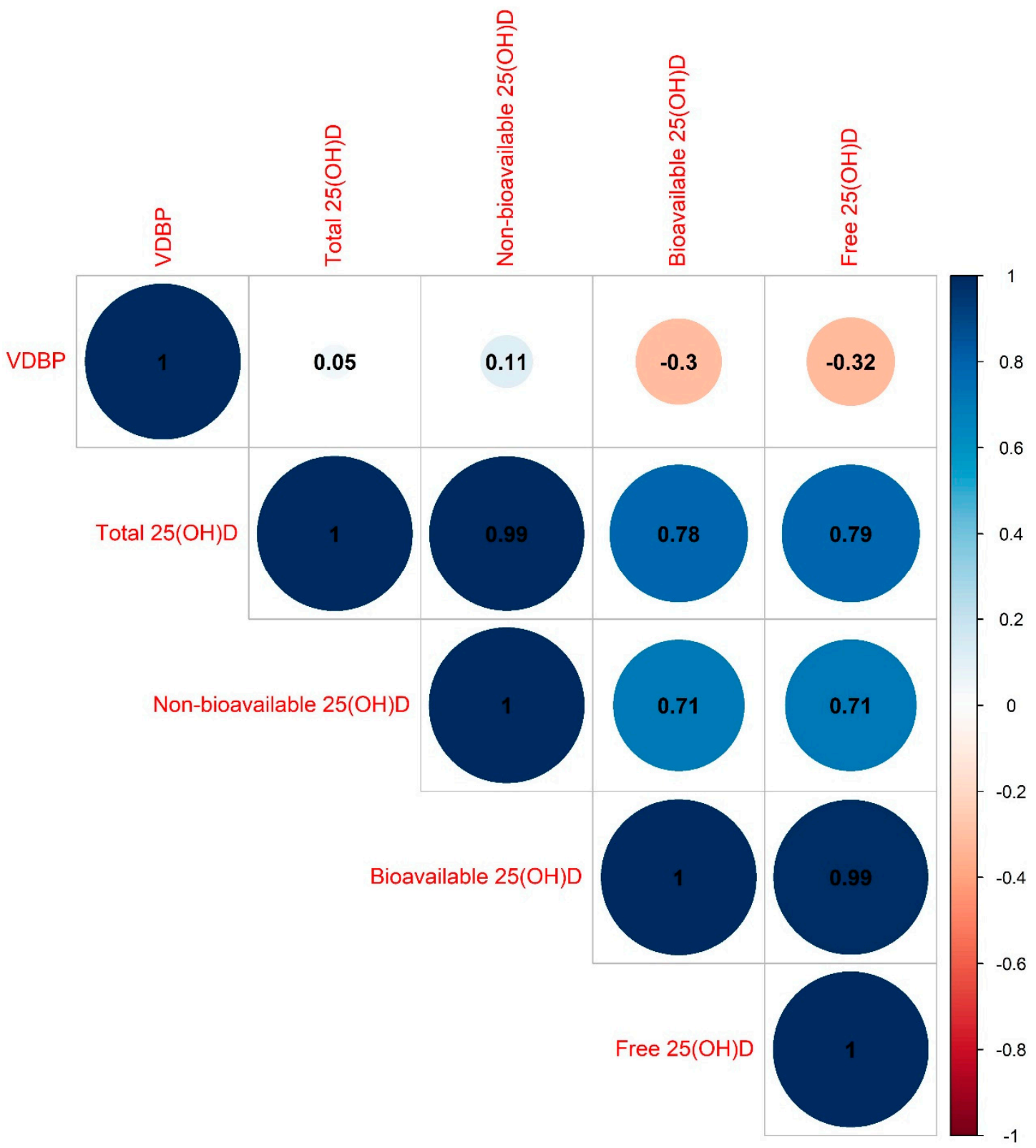

Figure 2. Correlation matrix between VDBP, total, non-bioavailable, bioavailable, and free 25(OH)D concentrations. Abbreviations: VDBP: vitamin D binding protein.

Figure 3 further plots the variations in VDBP, total, non-bioavailable, bioavailable, and free $25(\mathrm{OH}) \mathrm{D}$ concentrations by season and month of blood draw, and VDBP genotype. Seasonal variations were observed for all markers, with peak values in spring for VDBP (mean: $332.3 \mu \mathrm{g} / \mathrm{mL}$ ) and in summer for total, non-bioavailable, bioavailable, and free 25(OH)D (mean: $61.0 \mathrm{nmol} / \mathrm{L}, 52.9 \mathrm{nmol} / \mathrm{L}, 3.2 \mathrm{ng} / \mathrm{mL}$, and $7.1 \mathrm{pg} / \mathrm{mL}$, respectively). 
Additionally, the concentrations of vitamin D biomarkers differed by VDBP genotype. Participants with GC2-2 genotype had the lowest levels of VDBP (mean: $308.0 \mu \mathrm{g} / \mathrm{mL}$ ), total (mean: $46.8 \mathrm{nmol} / \mathrm{L}$ ), and non-bioavailable 25(OH)D (mean: $37.4 \mathrm{nmol} / \mathrm{L}$ ), but had the highest levels of bioavailable (mean: $3.7 \mathrm{ng} / \mathrm{mL}$ ), and free 25(OH)D (mean: $8.3 \mathrm{pg} / \mathrm{mL}$ ). Participants with GC1f-1f genotype had the lowest levels of bioavailable (mean: $1.4 \mathrm{ng} / \mathrm{mL}$ ), and free $25(\mathrm{OH}) \mathrm{D}$ (mean: $3.0 \mathrm{pg} / \mathrm{mL}$ ), but the highest level of non-bioavailable 25(OH)D (mean: $46.7 \mathrm{nmol} / \mathrm{L}$ ), and the second highest level of VDBP (mean: $332.4 \mu \mathrm{g} / \mathrm{mL}$ ), which was very close to those with GC1s-1s genotype (mean: $332.0 \mu \mathrm{g} / \mathrm{mL}$ ) and GC1f-1s genotype (mean: $332.6 \mu \mathrm{g} / \mathrm{mL}$ ). Those with GC1s-1s genotype had the highest level of total 25(OH)D (mean: $52.1 \mathrm{nmol} / \mathrm{L}$ ).

Table 2 presents VDBP, total, non-bioavailable, bioavailable, and free $25(\mathrm{OH}) \mathrm{D}$ concentrations by selected participant characteristics, and Table 3 presents results of the corresponding regression models in which all of the covariates were mutually adjusted for (in addition to adjustment by VDBP genotype and season of blood draw). VDBP levels decreased with increasing age and increasing BMI, and were lower in men and those with diabetes. By contrast, higher levels were observed among those reporting regular intake of vitamin supplements, with high total cholesterol and CRP levels. Both total and non-bioavailable $25(\mathrm{OH}) \mathrm{D}$ concentrations decreased with increasing age and increasing $\mathrm{BMI}$, and were lower among current smokers, and those with diabetes. However, higher concentrations were seen among males, those reporting more physical activity and regular intake of vitamin supplements. Non-bioavailable 25(OH)D concentrations were also higher among those with higher CRP levels. Bioavailable and free 25(OH)D concentrations decreased with increasing age and increasing BMI, and were lower among current smokers and those with high levels of total cholesterol. Lower bioavailable 25(OH)D levels were further seen among those with higher CRP levels. Higher concentrations of bioavailable and free $25(\mathrm{OH}) \mathrm{D}$ levels were seen among males, those reporting more physical activity and regular intake of vitamin supplements. 


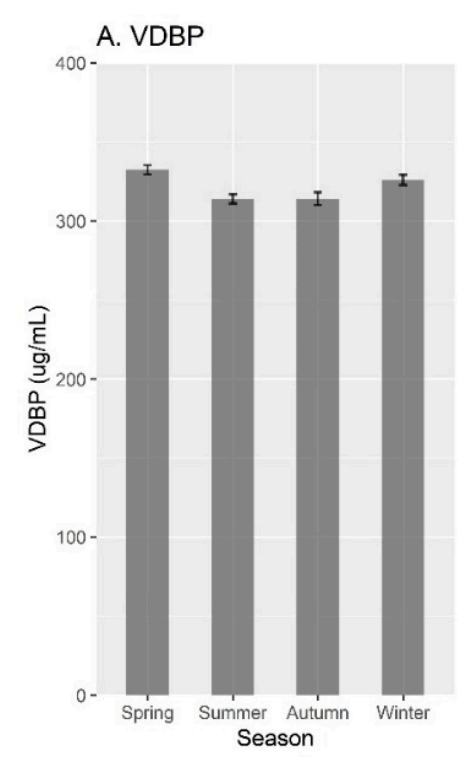

\section{B. Total $25(\mathrm{OH})$ D}
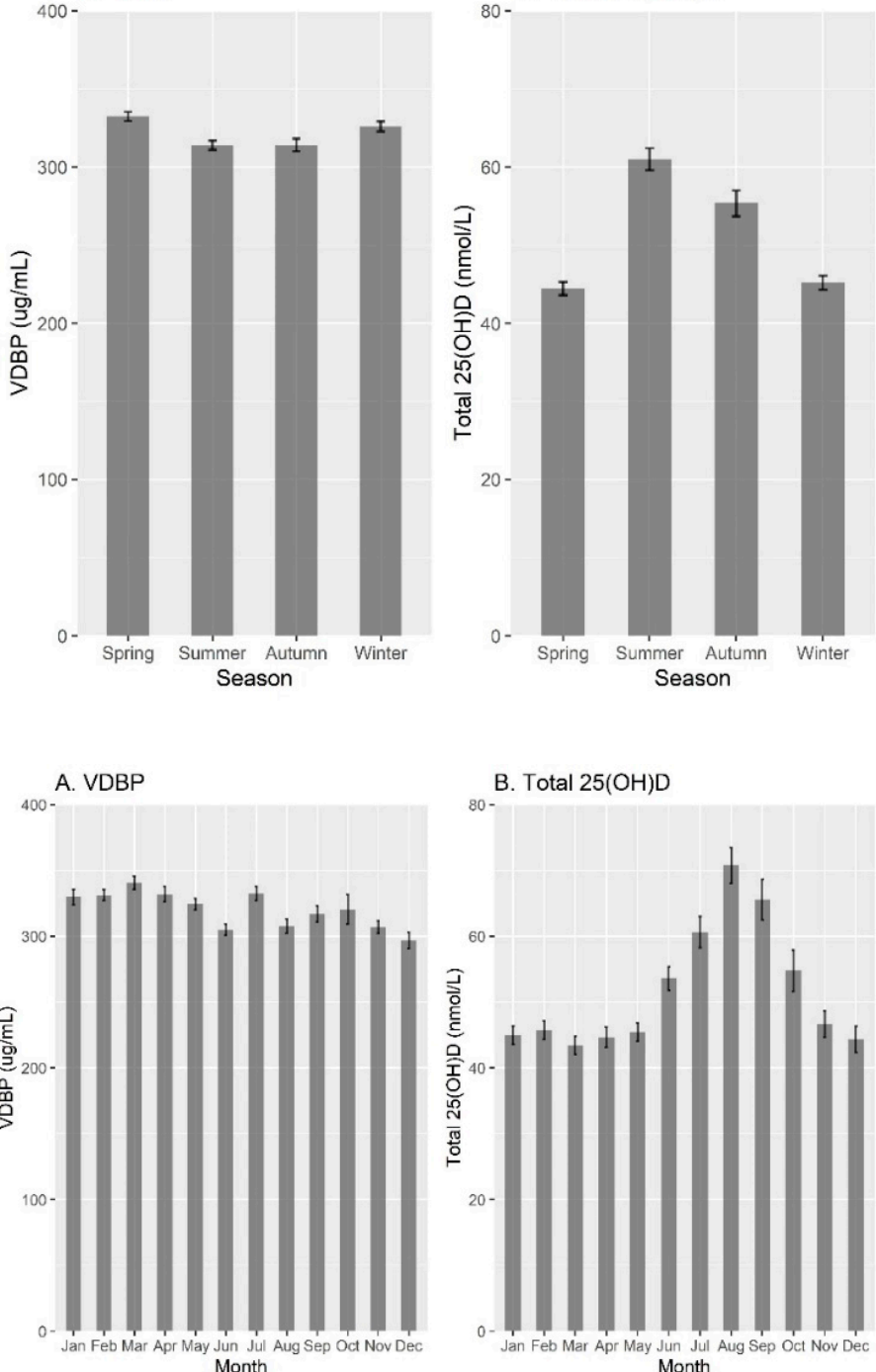

C. Non-bioavailable $25(\mathrm{OH}) \mathrm{D}$

D. Bioavailable 25(OH)D

\section{E. Free 25(OH)D}

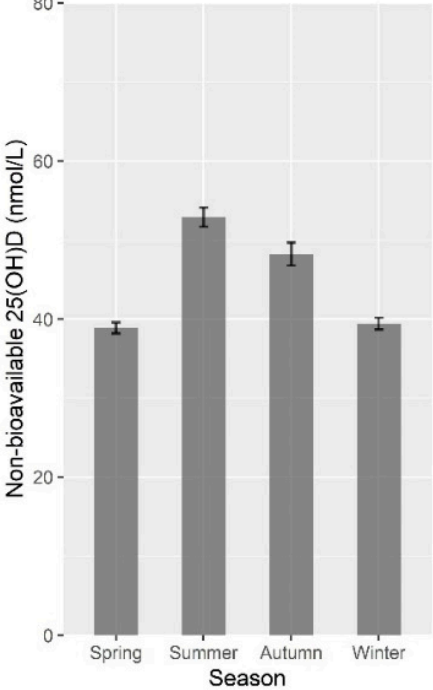

(a)

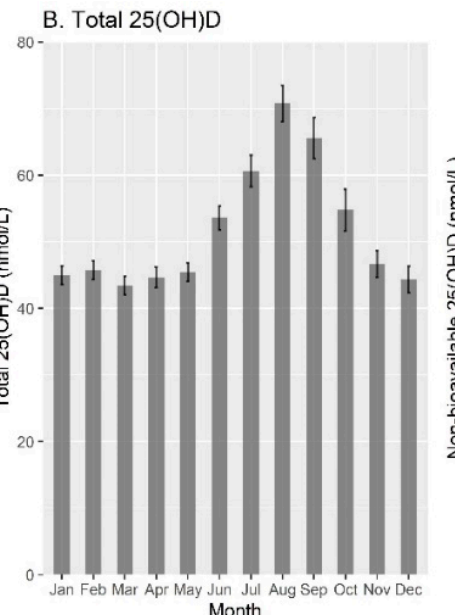

C. Non-bioavailable $25(\mathrm{OH}) \mathrm{D}$

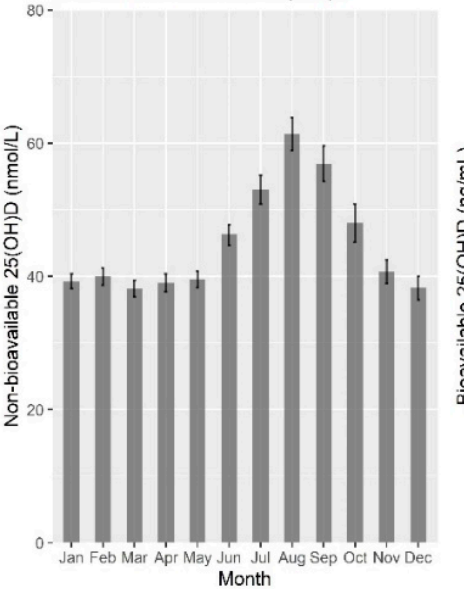

(b)

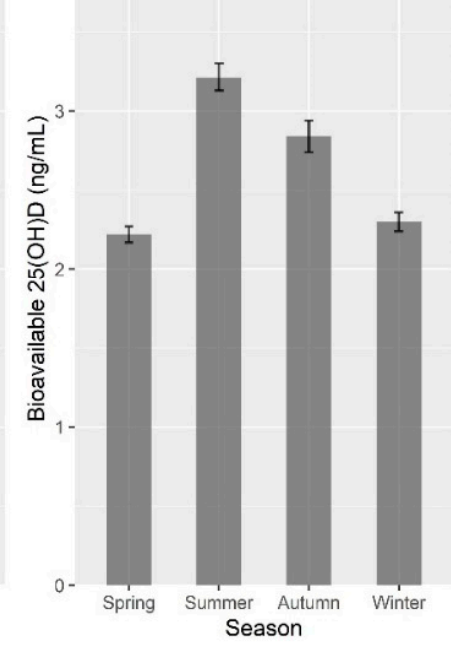

D. Bioavailable $25(\mathrm{OH}) \mathrm{D}$

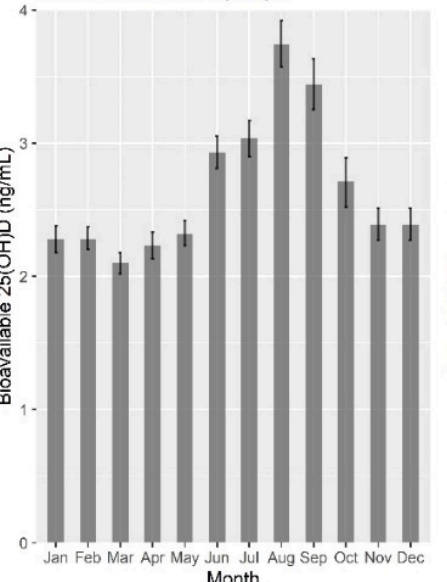

E. Free $25(\mathrm{OH}) \mathrm{D}$

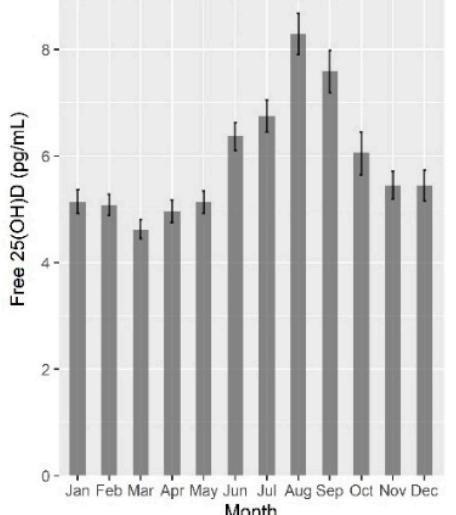

Figure 3. Cont. 

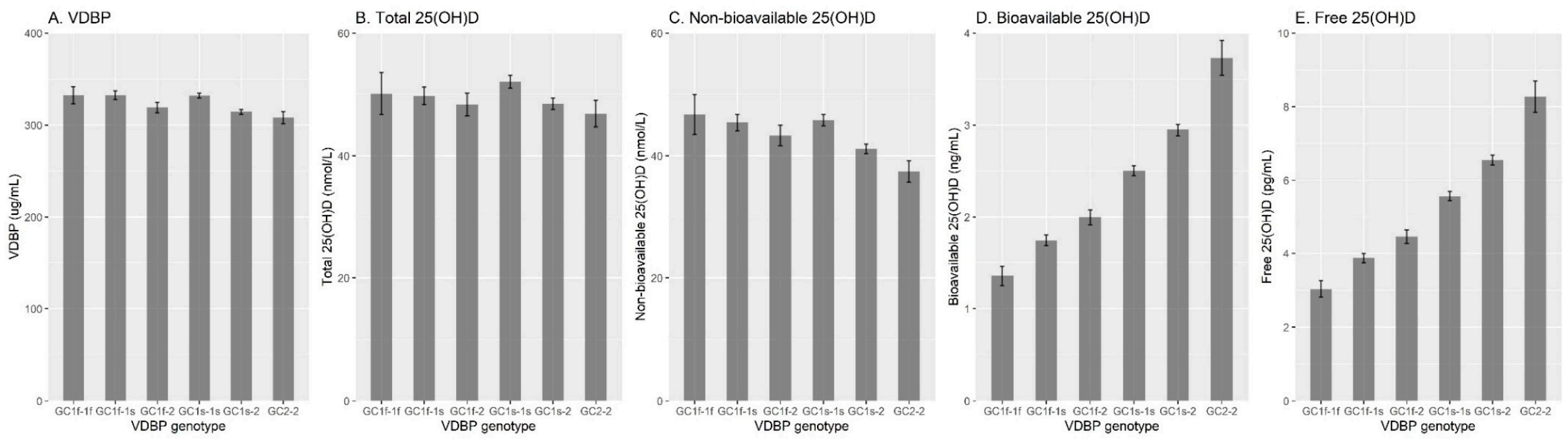

(c)

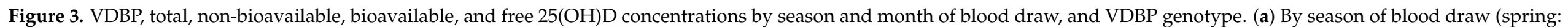

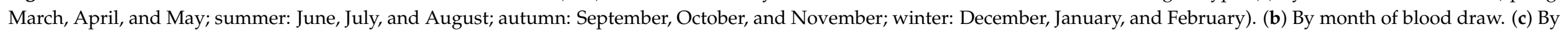
VDBP genotype. Abbreviations: VDBP: vitamin D binding protein. 
Table 2. VDBP, total, non-bioavailable, bioavailable, and free $25(\mathrm{OH}) \mathrm{D}$ concentrations by selected characteristics.

\begin{tabular}{|c|c|c|c|c|c|c|c|}
\hline \multirow[t]{2}{*}{ Characteristics } & \multirow[t]{2}{*}{ Group } & \multirow[t]{2}{*}{$\mathbf{N}$} & \multirow{2}{*}{$\begin{array}{c}\begin{array}{c}\text { VDBP } \\
(\mu \mathrm{g} / \mathrm{mL})\end{array} \\
\text { Mean (SE) }\end{array}$} & \multirow{2}{*}{$\begin{array}{c}\text { Total } \\
25(\mathrm{OH}) \mathrm{D} \\
(\mathrm{nmol} / \mathrm{L})\end{array}$} & \multirow{2}{*}{$\begin{array}{c}\text { Non-Bioavailable } \\
25(\mathrm{OH}) \mathrm{D}(\mathrm{nmol} / \mathrm{L})\end{array}$} & \multirow{2}{*}{$\begin{array}{c}\begin{array}{c}\text { Bioavailable } \\
\text { 25(OH)D } \\
(\mathrm{ng} / \mathrm{mL})\end{array} \\
\text { Mean (SE) }\end{array}$} & \multirow{2}{*}{$\begin{array}{c}\text { Free } \\
25(\mathrm{OH}) \mathrm{D} \\
(\mathrm{pg} / \mathrm{mL})\end{array}$} \\
\hline & & & & & & & \\
\hline All participants & & & $323.6(0.85)$ & $49.8(0.29)$ & $43.4(0.26)$ & $2.5(0.02)$ & $5.7(0.04)$ \\
\hline \multirow{4}{*}{ Age } & $<60$ years & 2229 & $329.5(1.43)$ & $51.3(0.52)$ & $44.8(0.46)$ & $2.6(0.03)$ & $5.7(0.07)$ \\
\hline & 60-64 years & 1664 & $324.6(1.57)$ & $51.2(0.57)$ & $44.6(0.50)$ & $2.6(0.03)$ & $5.8(0.07)$ \\
\hline & 65-69 years & 1267 & $320.1(1.72)$ & $48.4(0.56)$ & $42.2(0.49)$ & $2.5(0.04)$ & $5.6(0.08)$ \\
\hline & $\geq 70$ years & 739 & $314.8(2.33)$ & $46.4(0.72)$ & $40.3(0.62)$ & $2.4(0.05)$ & $5.5(0.11)$ \\
\hline \multirow{2}{*}{ Sex } & Females & 3310 & $332.0(1.16)$ & $46.1(0.30)$ & $40.3(0.26)$ & $2.3(0.02)$ & $5.1(0.04)$ \\
\hline & Males & 2589 & $312.9(1.20)$ & $54.6(0.53)$ & $47.3(0.46)$ & $2.9(0.03)$ & $6.4(0.07)$ \\
\hline \multirow{3}{*}{ School education } & $\leq 9$ years & 4429 & $324.2(1.00)$ & $49.1(0.33)$ & $42.7(0.29)$ & $2.5(0.02)$ & $5.6(0.05)$ \\
\hline & 10-11 years & 816 & $322.9(2.03)$ & $51.4(0.79)$ & $44.9(0.69)$ & $2.6(0.05)$ & $5.8(0.10)$ \\
\hline & $\geq 12$ years & 654 & $320.3(2.44)$ & $52.8(1.00)$ & $45.9(0.88)$ & $2.8(0.06)$ & $6.1(0.13)$ \\
\hline \multirow{3}{*}{ Smoking status } & $\begin{array}{c}\text { Never } \\
\text { smokers }\end{array}$ & 3005 & 325.1 (1.19) & $48.7(0.37)$ & $42.6(0.33)$ & $2.5(0.02)$ & $5.5(0.05)$ \\
\hline & $\begin{array}{l}\text { Former } \\
\text { smokers }\end{array}$ & 1918 & $319.5(1.52)$ & $53.4(0.56)$ & $46.4(0.49)$ & $2.8(0.04)$ & $6.1(0.08)$ \\
\hline & $\begin{array}{l}\text { Current } \\
\text { smokers }\end{array}$ & 976 & $326.9(1.94)$ & $46.1(0.72)$ & $40.1(0.64)$ & $2.3(0.04)$ & $5.2(0.10)$ \\
\hline \multirow{2}{*}{ Physical activity } & Low & 4013 & $323.5(0.99)$ & $47.8(0.34)$ & $41.7(0.30)$ & $2.4(0.02)$ & $5.4(0.05)$ \\
\hline & Moderate/high & 1886 & $323.8(1.60)$ & $54.0(0.56)$ & $47.0(0.49)$ & $2.8(0.04)$ & $6.2(0.08)$ \\
\hline \multirow{2}{*}{$\begin{array}{c}\text { Regular } \\
\text { multivitamin intake }\end{array}$} & No & 5043 & $322.4(0.88)$ & $49.4(0.32)$ & $43.0(0.28)$ & $2.5(0.02)$ & $5.6(0.04)$ \\
\hline & Yes & 856 & $330.9(2.66)$ & $52.3(0.77)$ & $45.8(0.68)$ & $2.6(0.05)$ & $5.8(0.10)$ \\
\hline \multirow{2}{*}{ Fish consumption } & $\begin{array}{c}<1 \\
\text { time/week }\end{array}$ & 1955 & $322.2(1.37)$ & $49.4(0.53)$ & $43.1(0.46)$ & $2.5(0.03)$ & $5.6(0.07)$ \\
\hline & $\begin{array}{c}\geq 1 \\
\text { time/week }\end{array}$ & 3944 & $324.3(1.07)$ & $50.0(0.35)$ & $43.5(0.31)$ & $2.6(0.02)$ & $5.7(0.05)$ \\
\hline \multirow{2}{*}{ Hypertension } & No & 3355 & $324.9(1.18)$ & $50.6(0.40)$ & $44.1(0.35)$ & $2.6(0.03)$ & $5.8(0.06)$ \\
\hline & Yes & 2544 & $321.8(1.20)$ & $48.7(0.43)$ & $42.5(0.37)$ & $2.5(0.03)$ & $5.5(0.06)$ \\
\hline \multirow{2}{*}{ Diabetes } & No & 5044 & $326.0(0.93)$ & $50.3(0.32)$ & $43.9(0.28)$ & $2.6(0.02)$ & $5.7(0.04)$ \\
\hline & Yes & 855 & $309.3(1.96)$ & $46.7(0.69)$ & $40.7(0.61)$ & $2.4(0.04)$ & $5.5(0.09)$ \\
\hline \multirow{2}{*}{$\begin{array}{l}\text { Cardiovascular } \\
\text { disease }\end{array}$} & No & 4739 & $325.5(0.95)$ & $50.0(0.32)$ & $43.6(0.28)$ & $2.5(0.02)$ & $5.7(0.05)$ \\
\hline & Yes & 1160 & $316.0(1.85)$ & $48.9(0.68)$ & $42.6(0.60)$ & $2.5(0.04)$ & $5.7(0.09)$ \\
\hline \multirow{2}{*}{ Cancer } & No & 5447 & $323.4(0.89)$ & $49.8(0.30)$ & $43.4(0.27)$ & $2.6(0.02)$ & $5.7(0.04)$ \\
\hline & Yes & 452 & $325.4(2.69)$ & $49.3(1.04)$ & $43.1(0.92)$ & $2.5(0.06)$ & $5.5(0.14)$ \\
\hline \multirow{2}{*}{$\begin{array}{c}\text { Chronic kidney } \\
\text { disease }\end{array}$} & No & 5405 & $323.7(0.88)$ & $49.9(0.30)$ & $43.5(0.26)$ & $2.6(0.02)$ & $5.7(0.04)$ \\
\hline & Yes & 494 & $322.2(3.12)$ & 48.5 (1.18) & $42.2(1.01)$ & $2.5(0.08)$ & $5.6(0.18)$ \\
\hline \multirow{4}{*}{ Body mass index } & $<25 \mathrm{~kg} / \mathrm{m}^{2}$ & 1619 & $331.3(1.68)$ & $51.5(0.58)$ & $45.0(0.52)$ & $2.6(0.03)$ & $5.8(0.08)$ \\
\hline & $\begin{array}{l}25-29.9 \\
\mathrm{~kg} / \mathrm{m}^{2}\end{array}$ & 2750 & $322.4(1.23)$ & $50.8(0.44)$ & $44.2(0.39)$ & $2.6(0.03)$ & $5.8(0.06)$ \\
\hline & $\begin{array}{l}30-34.9 \\
\mathrm{~kg} / \mathrm{m}^{2}\end{array}$ & 1188 & $319.3(1.86)$ & $47.2(0.57)$ & $41.2(0.50)$ & $2.4(0.04)$ & $5.4(0.08)$ \\
\hline & $\geq 35 \mathrm{~kg} / \mathrm{m}^{2}$ & 342 & $311.6(2.84)$ & $42.6(0.85)$ & $37.2(0.75)$ & $2.1(0.06)$ & $4.9(0.13)$ \\
\hline \multirow{2}{*}{ Total cholesterol } & $<200 \mathrm{mg} / \mathrm{dL}$ & 1345 & $310.5(1.68)$ & $51.8(0.65)$ & $45.0(0.57)$ & $2.7(0.04)$ & $6.1(0.09)$ \\
\hline & $\geq 200 \mathrm{mg} / \mathrm{dL}$ & 4554 & $327.5(0.97)$ & $49.2(0.33)$ & $42.9(0.29)$ & $2.5(0.02)$ & $5.5(0.05)$ \\
\hline \multirow{2}{*}{ C-reactive protein } & $<3 \mathrm{mg} / \mathrm{L}$ & 3619 & $317.7(1.03)$ & $50.6(0.37)$ & $44.0(0.33)$ & $2.6(0.02)$ & $5.8(0.05)$ \\
\hline & $\geq 3 \mathrm{mg} / \mathrm{L}$ & 2280 & $332.9(1.43)$ & $48.5(0.47)$ & $42.5(0.42)$ & $2.4(0.03)$ & $5.4(0.07)$ \\
\hline
\end{tabular}


Table 3. Associations of various characteristics with VDBP, total, non-bioavailable, bioavailable, and free $25(\mathrm{OH}) \mathrm{D}$ concentrations: results of multiple linear regression.

\begin{tabular}{|c|c|c|c|c|c|c|}
\hline \multirow[t]{2}{*}{ Characteristics } & \multirow[t]{2}{*}{ Group } & VDBP & $\begin{array}{c}\text { Total } \\
\text { 25(OH)D }\end{array}$ & $\begin{array}{c}\text { Non-Bioavailable } \\
\text { 25(OH)D }\end{array}$ & $\begin{array}{c}\text { Bioavailable } \\
\text { 25(OH)D }\end{array}$ & Free $25(\mathrm{OH}) \mathrm{D}$ \\
\hline & & Estimate (SE) & Estimate (SE) & Estimate (SE) & Estimate (SE) & Estimate (SE) \\
\hline \multirow{4}{*}{ Age } & $<60$ years & Ref & Ref & Ref & Ref & Ref \\
\hline & 60-64 years & $-4.54(2.14)$ & $-0.51(0.71)$ & $-0.46(0.62)$ & $-0.02(0.04)$ & $-0.01(0.09)$ \\
\hline & $65-69$ years & $-9.03(2.26)$ & $-3.27(0.74)$ & $-3.00(0.65)$ & $-0.11(0.04)$ & $-0.16(0.09)$ \\
\hline & $\geq 70$ years & $-14.88(2.61)$ & $-5.59(0.86)$ & $-5.10(0.75)$ & $-0.20(0.05)$ & $-0.33(0.11)$ \\
\hline \multirow{2}{*}{ Sex } & Females & Ref & Ref & Ref & Ref & Ref \\
\hline & Males & $-15.48(1.86)$ & $7.82(0.61)$ & $6.43(0.53)$ & $0.55(0.04)$ & $1.11(0.08)$ \\
\hline \multirow{3}{*}{$\begin{array}{l}\text { School } \\
\text { education }\end{array}$} & $\leq 9$ years & Ref & Ref & Ref & Ref & Ref \\
\hline & 10-11 years & $-4.21(2.43)$ & $1.51(0.79)$ & $1.26(0.70)$ & $0.10(0.05)$ & $0.18(0.10)$ \\
\hline & $\geq 12$ years & $0.09(2.68)$ & $-0.77(0.88)$ & $-0.74(0.77)$ & $-0.01(0.05)$ & $-0.08(0.11)$ \\
\hline \multirow{3}{*}{ Smoking status } & Never smokers & Ref & Ref & Ref & Ref & Ref \\
\hline & $\begin{array}{c}\text { Former } \\
\text { smokers }\end{array}$ & $2.13(2.04)$ & $1.28(0.66)$ & $1.12(0.58)$ & $0.06(0.04)$ & $0.16(0.08)$ \\
\hline & $\begin{array}{l}\text { Current } \\
\text { smokers }\end{array}$ & $0.05(2.50)$ & $-5.54(0.79)$ & $-4.84(0.70)$ & $-0.28(0.05)$ & $-0.57(0.10)$ \\
\hline \multirow{2}{*}{$\begin{array}{l}\text { Physical } \\
\text { activity }\end{array}$} & Low & Ref & Ref & Ref & Ref & Ref \\
\hline & Moderate/high & $1.98(1.79)$ & $3.33(0.59)$ & $2.88(0.52)$ & $0.18(0.03)$ & $0.38(0.08)$ \\
\hline \multirow{2}{*}{$\begin{array}{c}\text { Regular } \\
\text { multivitamin } \\
\text { intake }\end{array}$} & No & Ref & Ref & Ref & Ref & Ref \\
\hline & Yes & $6.87(2.37)$ & $3.84(0.78)$ & $3.50(0.68)$ & $0.14(0.05)$ & $0.32(0.10)$ \\
\hline \multirow{2}{*}{$\begin{array}{c}\text { Fish } \\
\text { consumption }\end{array}$} & $<1$ time/week & Ref & Ref & Ref & Ref & Ref \\
\hline & $\geq 1$ time/week & $2.39(1.80)$ & $0.43(0.59)$ & $0.42(0.51)$ & $0.01(0.03)$ & $0.03(0.07)$ \\
\hline \multirow{2}{*}{ Hypertension } & No & Ref & Ref & Ref & Ref & Ref \\
\hline & Yes & $0.99(1.71)$ & $-0.31(0.57)$ & $-0.25(0.50)$ & $-0.02(0.03)$ & $-0.10(0.07)$ \\
\hline \multirow{2}{*}{ Diabetes } & No & Ref & Ref & Ref & Ref & Ref \\
\hline & Yes & $-10.84(2.40)$ & $-2.40(0.79)$ & $-2.22(0.69)$ & $-0.07(0.05)$ & $-0.17(0.10)$ \\
\hline \multirow{2}{*}{$\begin{array}{l}\text { Cardiovascular } \\
\text { disease }\end{array}$} & No & Ref & Ref & Ref & Ref & Ref \\
\hline & Yes & $-1.29(2.16)$ & $-0.85(0.71)$ & $-0.74(0.62)$ & $-0.04(0.04)$ & $-0.10(0.09)$ \\
\hline \multirow{2}{*}{ Cancer } & No & Ref & Ref & Ref & Ref & Ref \\
\hline & Yes & $2.85(3.05)$ & $-0.18(1.01)$ & $-0.10(0.88)$ & $-0.03(0.06)$ & $-0.08(0.13)$ \\
\hline \multirow{2}{*}{$\begin{array}{l}\text { Chronic kidney } \\
\text { disease }\end{array}$} & No & Ref & Ref & Ref & Ref & Ref \\
\hline & Yes & $4.59(3.04)$ & $0.75(1.01)$ & $0.67(0.88)$ & $0.03(0.06)$ & $0.11(0.13)$ \\
\hline \multirow{4}{*}{$\begin{array}{l}\text { Body mass } \\
\text { index }\end{array}$} & $<25 \mathrm{~kg} / \mathrm{m}^{2}$ & Ref & Ref & Ref & Ref & Ref \\
\hline & $25-29.9 \mathrm{~kg} / \mathrm{m}^{2}$ & $-8.21(2.01)$ & $-1.69(0.66)$ & $-1.60(0.58)$ & $-0.04(0.04)$ & $-0.07(0.08)$ \\
\hline & $30-34.9 \mathrm{~kg} / \mathrm{m}^{2}$ & $-13.69(2.52)$ & $-4.63(0.83)$ & $-4.27(0.73)$ & $-0.14(0.05)$ & $-0.31(0.11)$ \\
\hline & $\geq 35 \mathrm{~kg} / \mathrm{m}^{2}$ & $-26.31(3.88)$ & $-8.92(1.28)$ & $-8.11(1.12)$ & $-0.32(0.07)$ & $-0.61(0.16)$ \\
\hline \multirow{2}{*}{$\begin{array}{c}\text { Total } \\
\text { cholesterol }\end{array}$} & $<200 \mathrm{mg} / \mathrm{dL}$ & Ref & Ref & Ref & Ref & Ref \\
\hline & $\geq 200 \mathrm{mg} / \mathrm{dL}$ & $13.19(1.97)$ & $-1.22(0.65)$ & $-1.00(0.57)$ & $-0.09(0.04)$ & $-0.34(0.08)$ \\
\hline \multirow{2}{*}{$\begin{array}{l}\text { C-reactive } \\
\text { protein }\end{array}$} & $<3 \mathrm{mg} / \mathrm{L}$ & Ref & Ref & Ref & Ref & Ref \\
\hline & $\geq 3 \mathrm{mg} / \mathrm{L}$ & $17.86(1.75)$ & $1.04(0.58)$ & $1.27(0.51)$ & $-0.09(0.03)$ & $-0.10(0.07)$ \\
\hline
\end{tabular}

Bold print indicates $p<0.05$. Abbreviations: SE: standard error; VDBP: vitamin D binding protein. The regression models were also adjusted for VDBP genotypes and seasons of blood draw. 


\section{Discussion}

In this large population-based cohort of older adults from Germany, total 25(OH)D levels were found to be low (mean: $49.8 \mathrm{nmol} / \mathrm{L}$ ), and to strongly vary by season of blood draw. Levels of non-bioavailable, bioavailable, and free 25(OH)D were strongly correlated with total 25(OH)D levels and showed similar major seasonal variation, with the highest concentrations in summer, and the lowest concentrations in spring. Despite strongly different absolute concentrations, all 25(OH)D measures also showed similar associations with potential non-genetic determinants, with decreasing levels with increasing age and BMI, and higher levels among males, non-smokers, and those reporting regular multivitamin intake. By contrast, VDBP genotypes showed opposite associations with bioavailable and free $25(\mathrm{OH}) \mathrm{D}$ (lowest levels among those with GC1f-1f genotype, highest among those with GC2-2 genotype), and non-bioavailable 25(OH)D (lowest among those with GC2-2 genotype, highest among those with GC1f-1f genotype). VDBP concentrations were highest in spring, lower in men than in women and unrelated to smoking, but they also strongly decreased with increasing age and increasing BMI. They showed moderate inverse correlations with bioavailable and free $25(\mathrm{OH}) \mathrm{D}$. In addition, some distinct patterns were seen, with markedly higher VDBP concentrations among those with high cholesterol and high CRP concentrations.

The associations of total $25(\mathrm{OH}) \mathrm{D}$ with potential determinants observed in our study are consistent with those observed in multiple previous studies and are meanwhile well established. This particularly applies to the strong seasonal variation caused by seasonal variation in ultraviolet-B exposure $[15,16]$, which is insufficient for effective synthesis of cholecalciferol in the skin during the winter months in Germany. Likewise, the strong decrease of $25(\mathrm{OH}) \mathrm{D}$ levels with increasing age as a result of decreasing ability of the skin to synthesize cholecalciferol and potentially less exposure to ultraviolet-B radiation is well established [9,17]. By contrast, evidence on potential determinants of specific components of $25(\mathrm{OH}) \mathrm{D}$ has been rather sparse and partly conflicting $[2,8,18,19]$. To our knowledge, ours is the first study to comprehensively assess associations of potential determinants with various components, including non-bioavailable, bioavailable, and free $25(\mathrm{OH}) \mathrm{D}$. The high positive correlations between those measures (and in particular between each of them and total $25(\mathrm{OH}) \mathrm{D}$ ) and the consistent patterns of associations with potential determinants (apart from genotypes) do not support suggestions for the need to evaluate different $25(\mathrm{OH}) \mathrm{D}$ components for assessing vitamin D status in clinical practice in an ethnically homogeneous population, such as our cohort, in which the vast majority of participants were Caucasians. Nevertheless, given the strong variation of bioavailable and free $25(\mathrm{OH}) \mathrm{D}$ by VDBP genotypes, and the strong variation of VDBP genotypes between ethnic groups $[13,14,20]$, different patterns might be expected and the role of different $25(\mathrm{OH}) \mathrm{D}$ components may be different in ethnically more diverse populations. Further research should address the associations of various $25(\mathrm{OH}) \mathrm{D}$ components with clinical outcomes in longitudinal studies in different types of populations.

The strong genetic determination of VDBP concentrations is well established $[13,20]$. Subjects with GC2-2 genotype have significantly lower VDBP concentrations than those with any other genotypes. In agreement with several previous studies [21,22], we found those with GC1f-1f genotype to have the highest VDBP concentrations. Different patterns were seen in the study by Powe et al. [13], where GC1f-1f was the most frequent genotype among Black Americans and associated with the lowest VDBP levels. Possible reasons for this apparent discrepancy, such as differences in antibodies used in the VDBP assays, or other factors and their possible clinical relevance, should be addressed in further research.

To our knowledge, our study is the first to comprehensively address potential nongenetic determinants of VDBP levels. Despite their strong genetic determination, quite pronounced inverse associations of VDBP levels were also seen with age and BMI, similarly to those seen for $25(\mathrm{OH}) \mathrm{D}$ concentrations. In contrast to the latter, however, VDBP concentrations were higher in women, and in participants with high cholesterol and CRP levels. The reasons for these strong associations are unclear and require further study. One 
possible explanation for the association observed with CRP levels might be an increase of VDBP concentrations in response to inflammation [23]. Our large study also disclosed small seasonal variations with peak values in spring in VDBP concentrations, which may previously not have been detected due to sample size limitations [15].

Our study has several strengths. To our knowledge, our study is the first one to provide a parallel comprehensive comparison of determinants of VDBP, total, bioavailable, and free $25(\mathrm{OH}) \mathrm{D}$ concentrations. Our study also has a much larger sample size than most previous studies assessing these various vitamin D related parameters. In addition to a range of genetic, lifestyle, and dietary factors, we simultaneously considered various health conditions reported by physicians in multivariable analyses. Furthermore, due to the population-based nature of our cohort, the generalizability of our findings for the older population may be higher than in studies focusing on specific patient groups.

Our study also has a number of limitations. Our analysis was limited to cross-sectional data, which limits any derivation of temporal or causal relationships. Although we have considered a large number of covariates in multivariable analyses, residual confounding might not be ruled out due to the observational nature of the study. We did not directly measure bioavailable and free $25(\mathrm{OH}) \mathrm{D}$, but determined it from total $25(\mathrm{OH}) \mathrm{D}$, VDBP, and albumin concentrations by previously suggested equations. Measured concentrations of free $25(\mathrm{OH}) \mathrm{D}$ have been reported to be lower than the calculated ones, especially under physiologic and pathologic conditions (15). Despite these limitations, the quite distinct associations and patterns observed in our study may stimulate further research towards a more complete understanding of the determinants and health relevance of VDBP and the various components of $25(\mathrm{OH}) \mathrm{D}$.

\section{Conclusions}

In this large population-based study of older adults from Germany, we found high correlations of total $25(\mathrm{OH}) \mathrm{D}$ with bioavailable, free, and non-bioavailable $25(\mathrm{OH}) \mathrm{D}$, along with quite consistent associations of these markers of vitamin $\mathrm{D}$ status with potential nongenetic determinants, despite quite diverse associations with VDBP genotypes. In addition, apart from their genetic determination, VDBP concentrations were found to strongly vary by a number of non-genetic potential determinants. The biological mechanisms and clinical implications of the observed patterns deserve careful elucidation in further research to better understand a potential role of vitamin $\mathrm{D}$ in its various compartments for health at older age.

Supplementary Materials: The following are available online at https:/ /www.mdpi.com/article/10 $.3390 /$ nu13113982/s1, File S1: Measurement and standardization of total 25(OH)D concentrations.

Author Contributions: H.B. and A.Z. conceived and designed the study. A.Z. conducted data analysis and drafted the manuscript. A.Z., S.K., T.N., B.H., B.S. and H.B. interpreted the results and revised the manuscript. All authors have read and agreed to the published version of the manuscript.

Funding: The ESTHER study was funded by grants from the Saarland state Ministry for Social Affairs, Health, Women and Family Affairs (Saarbrücken, Germany), the Baden-Württemberg state Ministry of Science, Research and Arts (Stuttgart, Germany), the Federal Ministry of Education and Research (Berlin, Germany) and the Federal Ministry of Family Affairs, Senior Citizens, Women and Youth (Berlin, Germany). The funders played no role in the design of the study, the collection, analysis, and interpretation of data; and in the decision to approve publication of the finished manuscript. The authors assume full responsibility for the analyses and interpretation of these data.

Institutional Review Board Statement: The ESTHER study was approved by the Ethics Committees of the Medical Faculty of the University of Heidelberg and of the Physicians' Board of Saarland.

Informed Consent Statement: All participants gave written informed consent.

Data Availability Statement: The data presented in this study are available on request from the corresponding author. The data are not publicly available due to privacy or ethical consideration.

Conflicts of Interest: The authors declare no conflict of interest. 


\section{References}

1. van Schoor, N.; Lips, P. Global Overview of Vitamin D Status. Endocrinol. Metab. Clin. N. Am. 2017, 46, 845-870. [CrossRef] [PubMed]

2. Yu, C.; Xue, H.; Wang, L.; Chen, Q.; Chen, X.; Zhang, Y.; Hu, G.; Ling, W. Serum Bioavailable and Free 25-Hydroxyvitamin D Levels, but Not Its Total Level, Are Associated With the Risk of Mortality in Patients With Coronary Artery Disease. Circ. Res. 2018, 123, 996-1007. [CrossRef]

3. Bikle, D.D.; Schwartz, J. Vitamin D binding protein, total and free Vitamin D levels in different physiological and pathophysiological conditions. Front. Endocrinol. 2019, 10,317. [CrossRef]

4. Bikle, D.D.; Malmstroem, S.; Schwartz, J. Current Controversies: Are Free Vitamin Metabolite Levels a More Accurate Assessment of Vitamin D Status than Total Levels? Endocrinol. Metab. Clin. N. Am. 2017, 46, 901-918. [CrossRef]

5. Tsuprykov, O.; Chen, X.; Hocher, C.-F.; Skoblo, R.; Yin, L.; Hocher, B. Why should we measure free 25(OH) vitamin D? J. Steroid Biochem. Mol. Biol. 2018, 180, 87-104. [CrossRef]

6. Goswami, R.; Saha, S.; Sreenivas, V.; Singh, N.; Lakshmy, R. Vitamin D-binding protein, vitamin D status and serum bioavailable 25(OH)D of young Asian Indian males working in outdoor and indoor environments. J. Bone Miner. Metab. 2016, 35, 177-184. [CrossRef]

7. Orces, C.; Lorenzo, C.; Guarneros, J.E. The Prevalence and Determinants of Vitamin D Inadequacy among U.S. Older Adults: National Health and Nutrition Examination Survey 2007-2014. Cureus 2019, 11, e5300. [CrossRef]

8. El Sabeh, M.; Ghanem, P.; Al-Shaar, L.; Rahme, M.; Baddoura, R.; Halaby, G.; Singh, R.J.; Vanderschueren, D.; Bouillon, R.; El-Hajj Fuleihan, G. Total, Bioavailable, and Free 25(OH)D Relationship with Indices of Bone Health in Elderly: A Randomized Controlled Trial. Clin. Endocrinol. Metab. 2021, 106, e990-e1001. [CrossRef] [PubMed]

9. Schöttker, B.; Hagen, L.; Zhang, Y.; Gào, X.; Holleczek, B.; Gao, X.; Brenner, H. Serum 25-Hydroxyvitamin D Levels as an Aging Marker: Strong Associations With Age and All-Cause Mortality Independent From Telomere Length, Epigenetic Age Acceleration, and 8-Isoprostane Levels. J. Gerontol. Ser. A Boil. Sci. Med. Sci. 2018, 74, 121-128. [CrossRef] [PubMed]

10. Löw, M.; Stegmaier, C.; Ziegler, H.; Rothenbacher, D.; Brenner, H. Epidemiological investigations of the chances of preventing, recognizing early and optimally treating chronic diseases in an elderly population (ESTHER study). Deutsch. Med. Wochenschr. 2004, 129, 2643-2647. [CrossRef]

11. Schöttker, B.; Jansen, E.H.J.M.; Haug, U.; Schomburg, L.; Köhrle, J.; Brenner, H. Standardization of Misleading Immunoassay Based 25-Hydroxyvitamin D Levels with Liquid Chromatography Tandem-Mass Spectrometry in a Large Cohort Study. PLoS ONE 2012, 7, e48774. [CrossRef] [PubMed]

12. Stocker, H.; Perna, L.; Weigl, K.; Möllers, T.; Schöttker, B.; Thomsen, H.; Holleczek, B.; Rujescu, D.; Brenner, H. Prediction of clinical diagnosis of Alzheimer's disease, vascular, mixed, and all-cause dementia by a polygenic risk score and APOE status in a community-based cohort prospectively followed over 17 years. Mol. Psychiatry 2020, 5, 1-11. [CrossRef]

13. Powe, C.E.; Evans, M.K.; Wenger, J.; Zonderman, A.B.; Berg, A.H.; Nalls, M.; Tamez, H.; Zhang, D.; Bhan, I.; Karumanchi, S.A.; et al. Vitamin D-Binding Protein and Vitamin D Status of Black Americans and White Americans. N. Engl. J. Med. 2013, 369, 1991-2000. [CrossRef]

14. Saarnio, E.; Pekkinen, M.; Itkonen, S.; Kemi, V.; Karp, H.; Kärkkäinen, M.; Mäkitie, O.; Lamberg-Allardt, C. Serum parathyroid hormone is related to genetic variation in vitamin D binding protein with respect to total, free, and bioavailable 25-hydroxyvitamin D in middle-aged Caucasians-A cross-sectional study. BMC Nutr. 2016, 2, 1. [CrossRef]

15. Oleröd, G.; Hultén, L.M.; Hammarsten, O.; Klingberg, E. The variation in free 25-hydroxy vitamin D and vitamin D-binding protein with season and vitamin D status. Endocr. Connect. 2017, 6, 111-120. [CrossRef]

16. Jablonski, N.G.; Chaplin, G. The roles of vitamin D and cutaneous vitamin D production in human evolution and health. Int. J. Paleopathol. 2018, 23, 54-59. [CrossRef] [PubMed]

17. Ferri, E.; Casati, M.; Cesari, M.; Vitale, G.; Arosio, B. Vitamin D in physiological and pathological aging: Lesson from centenarians. Rev. Endocr. Metab. Disord. 2019, 20, 273-282. [CrossRef] [PubMed]

18. Yang, L.; Chen, H.; Zhao, M.; Peng, P. Prognostic value of circulating vitamin D binding protein, total, free and bioavailable 25-hydroxy vitamin D in patients with colorectal cancer. Oncotarget 2017, 8, 40214-40221. [CrossRef]

19. Fang, A.; Long, J.; Zhang, Y.; Liu, Z.; Li, Q.; Zhang, D.; Luo, Y.; Zhong, R.; Zhou, Z.; Xu, Y.; et al. Serum Bioavailable, Rather Than Total, 25-hydroxyvitamin D Levels Are Associated With Hepatocellular Carcinoma Survival. Hepatology 2020, 72, 169-182. [CrossRef]

20. Bouillon, R.; Schuit, F.; Antonio, L.; Rastinejad, F. Vitamin D Binding Protein: A Historic Overview. Front. Endocrinol. 2020, 10, 910. [CrossRef]

21. Johnsen, M.S.; Grimnes, G.; Figenschau, Y.; Torjesen, P.A.; Almås, B.; Jorde, R. Serum free and bio-available 25-hydroxyvitamin D correlate better with bone density than serum total 25-hydroxyvitamin D. Scand. J. Clin. Lab. Investig. 2014, 74, 177-183. [CrossRef] [PubMed]

22. Sollid, S.T.; Hutchinson, M.Y.S.; Berg, V.; Fuskevåg, O.M.; Figenschau, Y.A.; Thorsby, P.M.; Jorde, R. Effects of vitamin D binding protein phenotypes and vitamin D supplementation on serum total $25(\mathrm{OH}) \mathrm{D}$ and directly measured free $25(\mathrm{OH}) \mathrm{D}$. Eur. J. Endocrinol. 2016, 174, 445-452. [CrossRef] [PubMed]

23. Kew, R.R. The Vitamin D Binding Protein and Inflammatory Injury: A Mediator or Sentinel of Tissue Damage? Front. Endocrinol. 2019, 10, 470. [CrossRef] [PubMed] 